\title{
RESEARCH ETHICS: A PRACTICAL GUIDE
}

\section{WHY IS THIS GUIDELINE IMPORTANT?}

Any research must follow ethical principles, particularly when it involves people as participants and is likely to impact them. This is standard practice in academic research and a legal requirement in medical trials, but also applies to research carried out by Oxfam. Oxfam's work focuses on vulnerable populations, and takes place under difficult circumstances - for example displacement, conflict or disasters from natural hazards. When research takes place in such vulnerable and fragile contexts, high ethical standards need to be met and tailored to the specific characteristics of each situation.

This guideline takes these complexities into account and sets out the minimum ethical standards required in all research conducted, managed or commissioned by Oxfam. It therefore applies to all Oxfam staff and partners, including consultants and/or contracted researchers and enumerators (referred to as 'researchers' in this document, for simplicity).

This guideline is based in part on the UK Economic and Social Research Council (ESRC) Framework for Research Ethics that is used by social science academics in the UK. There are important additions, in line with Oxfam's commitment to decolonizing research and recent literature that highlights additional issues for ethical research standards in development research (McDermott et al. 2019). Moreover, because Oxfam operates as a large confederation of national organizations, researchers must adhere to any context-specific and more detailed guidance or protocols provided by affiliates or partners.

Oxfam welcomes the adaptation of this guideline by other NGOs, community organizations and researchers working in fragile contexts and with vulnerable communities. The guideline should be read together with other relevant Oxfam and Oxfam GB policies and protocols (as specified throughout the document), including the guidelines on Writing Terms of Reference for Research (with the accompanying Terms of Reference for Research Template), Integrating Gender in Research Planning and Doing Research with Enumerators. 


\section{RESEARCH ETHICS}

\section{What are research ethics?}

Research ethics are a set of principles and guidelines that shape and guide the way any research involving sentient beings (i.e. people and animals) is designed, conducted, managed, used and disseminated. In these guidelines, the term 'research' is used broadly: it includes diagnostic and explorative investigations of social issues of interest, as well as work carried out as part of Monitoring, Evaluation, Accountability and Learning (MEAL) mandates focusing on specific efforts (intervention, policy, programme).

\section{What are the core principles of research ethics?}

The six key principles for ethical research, as outlined by the ESRC, are:

- research should aim to maximize benefit for individuals and society, and minimize risk and harm;

- the rights and dignity of individuals and groups should be respected;

- $\quad$ wherever possible, ${ }^{1}$ participation should be voluntary and appropriately informed;

- research should be conducted with integrity and transparency;

- lines of responsibility and accountability should be clearly defined; and

- independence of research should be maintained and, where conflicts of interest cannot be avoided, they should be made explicit.

In addition, in line with Oxfam's mission of working towards a just world without poverty - and recognizing the privileged position of power and trust that Oxfam holds in relation to the communities it serves - ethical research must:

- challenge any form of harassment, discrimination, intimidation, exploitation or abuse; and

- not allow the abuse of positions of power and unequal power relationships in any way.

In order to ensure that these principles guide any research conducted by or for Oxfam, it is necessary to comply with the standards and procedures set out in these guidelines throughout the lifecycle of research projects.

\section{Research ethics in practice}

Oxfam's research often takes place in the Global South, in settings characterized by poverty; shrinking civic space or limited rights infrastructure; fragility or insecurity; and often under the watch of repressive governments. Not only do such conditions make the need for ethical research standards even clearer, they also affect the way these standards should be implemented, and make them highly context-specific. The ownership of local staff in upholding ethical research standards is paramount.

\footnotetext{
${ }^{1}$ As the ESRC clarifies, in some cases, it might not be possible to gain informed consent from participants before they are involved in research - for example, research involving large groups (e.g. a study of street protests) or experimental studies, where disclosing detailed information would alter participants' behaviours. Covert or deceptive research should not be used routinely or lightly, and should only be undertaken when no other approach is possible.
} 
The following standards should be adhered to throughout the lifecycle of research projects.

\section{Designing ethical research}

- Research must be designed with the aim to maximize benefits for participants, for example to improve working conditions in a garment factory. Potential benefits must always outweigh potential risks.

- It is Oxfam's duty to inform respondents of the research objectives and methods, as well as how and in what format they can expect to learn about the findings.

- Any staff carrying out research on behalf of Oxfam must be qualified and trained for the task. Recruitment of researchers must be planned thoroughly.

- Research methods and tools (e.g. survey/interview questions, focus group activities, etc.), and training and workshops for local research staff must be context-specific as well as gender- and socio-culturally sensitive.

\section{Conducting and signing off a risk analysis}

- Once a research plan exists, the project cannot start until a risk analysis is conducted and signed off by all relevant central and local team members. These are typically the local programme and/or advocacy teams, in conjunction with the research lead.

- Risk analyses aim to reduce the potential for unintended harm. All potential risks must be identified and recorded, and a risk mitigation strategy proposed. A risk analysis needs to be continuously updated throughout the course of a research project. If it emerges that the research carries a higher risk for any one group or person than was initially thought, then it should be redesigned to address and reduce those risks. If this is not possible, the research cannot go ahead.

- A risk analysis must differentiate between short-term risks (for example, immediate dangers that could arise during the fieldwork) and long-term risks (for example, participants' future eligibility for programme benefits). It must also consider risks to researchers (see Acknowledging researchers' roles), in line with Oxfam's duty of care towards staff.

- A risk analysis needs to include considerations of the potential need for researchers to limit participants' confidentiality (i.e. disclose their identity) for safety or legal reasons (see Confidentiality and anonymity). This includes an exploration of what could potentially lead to limiting confidentiality; the identification of a protocol to follow in these cases, including signposting and reporting procedures; and contact details of a safeguarding focal point.

- The names and contact details of both research and safeguarding focal points should be clearly stated in the risk analysis (see Informed consent).

\section{Selecting participants}

- Participants should only be involved in research that has some potential benefit for them. Possible outcomes, such as a safer society or better working conditions in the long run, may be benefits if the individual participants consider them to be so. Some participants may feel a benefit simply from having the chance to tell their story. However, it is up to them to decide whether this is the case.

- To ensure participants can make an informed judgement about what potential benefits the research in question may bring to them, they need to properly understand its purpose. It is important to remember that they may expect a benefit (such as in-kind or material assistance) in return for participating.

- Be aware of settings in which there is a risk of research fatigue, i.e. multiple pieces of research having taken place in parallel or close together with little tangible gain for communities (Way, 2013). This could entail a higher chance of bias in responses, because respondents cannot see a clear benefit to participating in another piece of research. 


\section{Gaining the consent of participants}

- Researchers must gain informed and voluntary consent from participants before conducting research. This means that participants should receive all relevant information about the research and be able to understand it; be free to choose whether or not to participate and to withdraw from the research at any time; and be provided with ongoing access to means by which to express concerns. The depth of this consent-taking process will depend on the topic and the extent to which the research could impact on the participants' lives.

- If research involves children, as defined by national law, then their parents or guardians must also give consent. It is best to get their written consent, in case of disputes later. Care should also be taken to ensure that the information given about the research is age-appropriate.

- Special care must be taken when seeking consent from particularly vulnerable groups, for example prisoners or survivors of sexual abuse.

- Researchers must ensure that no participants are forced to take part, for example by their employer, parents, community leaders or elders.

- Participants in research should understand and agree to how Oxfam would like to use the research findings and if/to what extent Oxfam may refer to personal information in the process.

Further guidance on these topics is provided in the Informed consent and Confidentiality and anonymity sections.

\section{Conducting research}

- Oxfam must provide adequate training for researchers, so they fully understand the Code of Conduct they are required to sign.

- Oxfam must monitor safe research practices (see Monitoring safe research practices and the guidelines on Doing Research with Enumerators).

- Enumerators should be trained not solely on the research to be conducted, but also what it means to be the first point of contact for community members in difficult situations, and where NGOs might already operate below acceptable standards (e.g. misconduct, safeguarding issues, abuse of power, etc.).

- Research should be conducted in places that are as safe and socially comfortable for both participants and researchers as possible - and where respondents are able to speak as freely as possible.

- If participants have incurred direct financial costs, they can be reimbursed through tokens such as vouchers or a context-appropriate gift proportional to the amount of time spent on the research. Oxfam does not generally recommend financial compensation, as this could be considered a form of coercion to take part in research. However, this is highly contextdependent and local staff will know best what compensation is appropriate. For example, when research takes up time respondents would have otherwise spent doing paid work, then payment at a reasonable rate based on local income-per-time calculations can be considered ethical. It may also be appropriate to reimburse with mobile phone credit, especially for phone interviews.

- The participants must be able to contact the researchers, whether directly, through local partners or other ongoing routes of access. Part of obtaining informed consent includes making sure that participants understand they are free to make contact at any point.

- If a participant reports any serious adverse effects as a result of participating - such as losing their job or being physically abused - this must be reported to the research or safeguarding focal points. If these adverse effects have resulted from research staff misconduct, internal reporting procedures should be followed (see Acknowledging researchers' roles), as appropriate. 


\section{Using research findings}

- Data obtained from ethical research has to be treated confidentially and stored safely, in line with internal data protection standards and any relevant local or national privacy or information rights law (see Data protection). Many of the countries in which Oxfam operates have their own data protection and privacy standards.

- Even after the research is concluded, participants must be able and feel sufficiently comfortable to contact Oxfam's research focal point to enquire about the outcomes.

- Data are normally stored, analysed and reported in an anonymized format. If it is agreed that all or any part of a participant's testimony can be used publicly without anonymization by Oxfam, this commitment must be clearly recorded and respected, as well as fully riskassessed. Children should never be identified, even with their or their guardians' consent (see Confidentiality and anonymity).

\section{INFORMED CONSENT AND MANAGING DATA}

\section{Informed consent}

A participant's ability to understand the purpose of the research is integral to truly informed consent, as is their ability to opt out, and raising doubts or questions. The process of gaining informed consent is pervaded by power imbalances between researchers, participants and other stakeholders. It is the responsibility of those carrying out the research to address and minimize these power differences as much as possible, including by making them explicit in the risk assessment.

To ensure that people are able to make an informed decision about their participation in research, it is paramount to provide participants with sufficient information on the research project. They should also be able to refer back to this information, for example through information sheets handed out before the start of the research, and to do so safely (e.g. when the topic of enquiry is particularly sensitive, such as gender-based violence).

Information to prospective participants should detail:

- who and which organization(s) are collecting their data and carrying out the research;

- what the study is about;

- which activities participants will be involved in (e.g. interviews, focus groups, surveys);

- how data will be captured (e.g. audio or video recordings, online or paper surveys);

- how the data will be stored and with whom - and in which countries - the data will be shared;

- how the findings from the research will be used (e.g. policy, media or academic outputs);

- how confidentiality and anonymity will be preserved, as required, and what circumstances might require confidentiality to be breached (see Limits to confidentiality); and

- how to withdraw from the research, complain or express concerns.

It should also emphasize that participation is voluntary.

Contact details should be given for the following focal points:

- Research: for example, to ask how the research will be used or how the findings can be accessed in the future. This focal point must coordinate with the information security team if there are issues related to the use of personal data, and with the safeguarding team if there are any safeguarding concerns.

- Safeguarding: for example, to alert the team that staff are acting in conflict with the Code of Conduct. If internal mechanisms are available, relevant contact details should be provided. 
For example, Oxfam GB's safeguarding team can be emailed at safeguarding@oxfam.org.uk and SpeakUp@oxfam.org.uk, and a misconduct webform is available at https://oxfam.cluewebforms.co.uk/webform/misconduct/en. If other NGOs or independent researchers do not have access to an internal safeguarding team, the contact details of staff not directly involved in the research project should be provided, so participants can raise any concerns about the research team.

- Data Protection: for example, to express or escalate concerns about privacy. It may be appropriate to include a local focal point (teams may have a Data Protection Focal Point), but the contact details of the organization or organizations' Data Protection officer should always also be provided. For example, Oxfam GB's Privacy Team can be emailed at privacy@oxfam.org.uk.

Contacting these focal points should be possible through more than one avenue (e.g. in person, email, webform and phone number) - consideration should be given in the risk analysis to ease of contact and agency.

It is important to remember that informed consent means that:

- Participants are free to give consent and to withdraw at any time without detriment to themselves or their present/future involvement with Oxfam and partners;

- Consent is given voluntarily, i.e. no pressure or coercion must be applied, and no rewards of any kind promised in exchange;

- Particular attention should be paid when researching vulnerable groups with low agency, for example, children or minors, people living with disabilities or mental illness, prisoners, ethnic minorities and displaced people;

- Information should be provided in a way that is accessible to the groups researched, for example, through the use of images, age-appropriate language and translations; and

- Consent is not a simple one-time transaction; rather, it might need to be re-negotiated over time, particularly when conducting longitudinal studies, to ensure that any changes in the research are communicated and participants have the chance to withdraw.

Informed consent must be recorded either:

- In writing (by signing or ticking a consent form) - this is preferred but not always possible; or

- Verbally, which should be witnessed and audio-recorded, for example on a tablet or phone. This may be obtained for instance, before key informant interviews or when respondents are illiterate.

It is crucial to monitor that informed consent is obtained following these standards throughout data collection. This must be done either by in-person supervision or through an intermediary, and in the case of digital data collection it can be supported by automated data checks that, for example, measure the time spent on obtaining consent by an enumerator.

For further guidance and templates for recording informed consent, see these examples from the World Health Organization, the University of Manchester, the University of Michigan, the UK Data Service and Oxfam's Ethical Content Guidelines.

\section{Confidentiality and anonymity}

Confidentiality in social research is about researchers not disclosing any identifiable information that might lead others to recognize research participants. It entails careful management of data (i.e. storing data securely and separating identifiable information from other data), and ensuring 
that the same standards maintained by all those who access any personal information (e.g. research team members and transcribers).

Anonymization of data is a step taken by researchers to help protect participants' identities by removing any identifiable information. It is a powerful tool to reduce risk of harm to individuals. Consideration should be given in the risk analysis to how robust anonymization can be, as well as whether community-level data may still produce risk of harm (for instance, if it identifies vulnerable LGBTQ+ or ethnic groups in a population).

It is worth noting that the right to withdraw personal information only applies to identifiable data, not data that have been fully anonymized, i.e. when it is no longer possible to recognize a participant. Participants should be given a deadline by which it will be possible to withdraw from the research (i.e. before data is anonymized), along with a clear timeline of when personal information and other identifiable data will be deleted.

It is essential that the data collected are treated confidentially, and stored securely and as anonymously as possible. It is paramount that identifying information is only known to the research team and not disclosed publicly, unless it was explicitly agreed with participants during the consent process - for example, for illustrating publications with first-hand narratives.

It is important to note that participants must always be asked, must be free to choose and must agree on whether or not:

- they can be quoted anonymously in Oxfam materials - the most common approach taken when presenting research findings;

- their real name can be used in Oxfam materials; and/or

- any photographic images and/or video recordings of them can be used in Oxfam materials. This aligns with Oxfam's Digital Safeguarding Policy and Oxfam's Ethical Content Guidelines.

Note: children must never be identified, in line with Oxfam's Youth Safeguarding Policy.

In practice, there are three 'levels' of stored data:

- Personal information. This includes names, phone numbers, photos and addresses.

- Pseudonymized data. This means direct identifiers have been removed, but it is possible to re-identify a participant from reference numbers, or a combination of other information included in the data, locations or detailed accounts (e.g. interview transcripts).

- Fully anonymized data. This means all identifiers have been removed, so that reidentification is highly unlikely given the anticipated use.

The exact level of security needed in storing data should be proportional to the risk it could pose if accessed by the wrong person. However, all researchers should always:

- Restrict access to raw data or findings to the research team and authorized persons only; and

- Store data on approved platforms or servers, which usually precludes most file-sharing platforms as well as personal computers and any unencrypted device (e.g. USB sticks). If the research involves the use of audio-recorders, encrypted devices should be used.

Whenever research findings or data are shared, for example during analysis, they must be either pseudonymized or fully anonymized. To do this, it is necessary to:

- Separate data from any personally identifiable information;

- Restrict sharing combinations of information that could be used to re-identify individuals;

- Omit all personal and identifiable information that would allow re-identifying participants, including in combination with publicly available data (full anonymization); 
- Consider what other information would be available with which people outside the research team might make or infer links beyond the data provided (for instance, if disaggregated data makes identifying a small pool of people or an individual likely); and

- Ensure a checkpoint is built into the process at which research outputs are reviewed and an attempt is made to 'falsify' the strategy by trying to re-identify the data.

There are various techniques that can be used to anonymize data - for example, the UK Data Service offers helpful anonymization protocols.

\section{Limits to confidentiality}

It is recognized that there might be some limits to confidentiality:

- To honour our duty of care, for example when there is danger to participants or researchers (which, when relevant, they themselves can raise through safeguarding and whistleblowing mechanisms);

- If there are other legal obligations or matters of public interest, for instance in preventing crime, safeguarding staff, court proceedings, or potentially if compelled by a legal process; and

- When data are about very specific topics and experiences that might make subjects recognizable despite anonymization, for example interviews with experts or reports about political leaders. This non-anonymized information-sharing needs to be explained and agreed to by participants during the informed consent process.

When becoming aware of safeguarding issues and hence potentially considering breaching confidentiality:

- It is crucial to assess the risk and harm of reporting or not reporting to both participants and researchers. Oxfam's safeguarding team should always be contacted before any actions are taken, so that a complete risk assessment can be carried out involving the people who are reporting the issue.

- Participants must be consulted and informed before any reporting is initiated - breaching confidentiality must never happen without this consultation. It is also possible for reporting to be done anonymously (see next point).

- Participants have the right to refuse to report a safeguarding issue and take a complaint forward with their identity disclosed. In this instance, the issue should still be reported to Oxfam's safeguarding team with no identifying information, so they can take this forward anonymously and work on the general issue raised by introducing mitigations to reduce risks. The team would always try to work with reporters, but reporters have the right to refuse engagement.

- Exceptional circumstances would involve child abuse or if others are at risk of significant harm if no action is taken. In such cases, Oxfam's safeguarding team would work with the complainant to keep them safe and anonymous while taking relevant action. Researchers should also contact their Data Protection Officer where they have serious ethical/legal concerns about sharing or using data in specific contexts.

If researchers become aware of safeguarding concerns that could prompt limiting confidentiality, they should work closely with participants and Oxfam's safeguarding team to mitigate potential harm, and assess whether the risks represent obstacles to conducting the research safely.

Note: Risks to confidentiality and anonymity in the research process - including limits or potential avenues of disclosure - must be acknowledged in the risk assessment and explained to participants as part of the Informed Consent process. 


\section{Data protection}

There is an explicit right to privacy (i.e. the universal human right to be in control of information about one's self, including who can process their data and for what purpose) in the UN Declaration of Human Rights. Within the EU, it is outlined in Article 8 of the EU Charter of Fundamental Rights.

This right is codified in law in many national and supranational conventions and legal frameworks referred to as 'Data Protection law'. Privacy/Data Protection principles are generally designed to protect individuals' agency when data about them is used, as well as requiring accountability for organizations, and reducing or preventing harm.

Data protection and privacy obligations are likely to come from three main places:

1. The law in the countries where Oxfam is incorporated or has a primary registration. In the EEA, this mainly means the EU General Data Protection Regulation (GDPR), and in Oxfam GB its implementation in UK law (the Data Protection Act 2018).

2. The law in the countries where data is being collected. There are a variety of legal frameworks applying to data internationally.

3. Oxfam's internal policies and frameworks, which can include requirements aligned with these frameworks, such as Oxfam GB's Data Protection Policy and Responsible Data policy.

There are many areas in which privacy principles and data protection law overlap with and amplify the principles of research ethics. Many of the recommendations in these guidelines - in particular, on confidentiality, risk, consent and accountability - if followed, will also ensure compliance with privacy principles.

A general focus on risk and harm is a key theme of most data protection legislation; if these are well considered in the research planning, it is more likely that the research will be substantially safe and compliant.

Researchers need to identify any specific restrictions or requirements around different types of data and activities and relevant local laws, as well as obtain appropriate advice from the organization's Data Protection Officer/Focal Point, or an appropriate legal adviser.

\section{SAFETY DURING RESEARCH}

\section{Monitoring safe research practices}

An upfront risk analysis is incomplete without monitoring ongoing research (Grimm et al, 2020). Both processes need to be steeped in local experience. If, during research, it becomes clear that harm is being caused to participants or researchers, the research must be reviewed and, if needed, stopped immediately. This is particularly important when working with vulnerable participants, in highly repressive or fragile contexts, and/or while researching sensitive topics.

The research information sheet used to seek informed consent should ensure that participants and researchers alike know how to contact the research, safeguarding and data protection focal points to report concerns and issues at any time.

Oxfam GB has comprehensive safeguarding policies, all of which apply to research of any kind, no matter the context. Again, all staff and partners should be familiar with these, or the equivalents in their own offices and organizations, and ensure that research is informed by them. 
This also applies to policies relating to conduct at work and sexual misconduct, such as Oxfam's Staff Code of Conduct, which all researchers need to sign.

Monitoring research practices to ensure these policies are upheld and lived, rather than just signed upfront, is Oxfam's standard. This can be done by ensuring in-depth discussion and training (beyond a simple signature) on all the relevant policies for everyone involved in research projects.

\section{Acknowledging researchers' roles}

Oxfam has the same duty of care towards researchers as it does towards participants. Risks posed to researchers should therefore be assessed, and adequate strategies put in place to mitigate them. There may also be instances in which researchers, inadvertently or knowingly, do not uphold the highest standards of research ethics or fully follow Oxfam's Code of Conduct.

Risks to researchers can originate from a range of sources, including:

- Unsafe working environments, with risks of physical threat or abuse;

- Internalizing the emotional burden of participants, sometimes involving emotional and psychological trauma;

- The potential that participants report situations of danger and abuse to researchers;

- Tight budgets and schedules that create untenable working conditions, such as overtime and work during local or religious and public holidays; and

- Increased exposure to the risks of everyday life and social interactions, especially for researchers that are part of the community in question.

To mitigate as many of these risks as possible:

- The risk analysis conducted before starting the research must make explicit and detailed reference to the context in which researchers work, mitigation strategies must be proposed and the ongoing research must be monitored;

- Emotional support should be made available to researchers through regular contact with team members and line managers, as well as signposting to Oxfam affiliates' staff health resources, if available;

- Training for researchers should cover how to access this support, how to listen and act when being confronted with experiences of trauma and abuse, and how to make a report to Oxfam's safeguarding team if needed; and

- A 'buddy system' can be used to monitor and increase researchers' physical safety researchers work in pairs, with regular check-ins arranged with each other. If one of the researchers cannot be contacted at the agreed time or within a reasonable period, depending on the circumstances, the matter can be escalated using a risk-based escalation process established in advance.

If it becomes clear that research involves serious harm to researchers, it must be stopped and any immediate danger promptly addressed and resolved, so as to guarantee that researchers are safe, whether the research resumes or not.

There might also be instances in which researchers' conduct breaches research ethics standards - for example, when they inadvertently cause psychological or physical harm to others through the content or context of the research, or do not follow adequate procedures to obtain informed consent, for instance, due to time pressure. Again, to mitigate these risks, an upfront risk analysis must be carried out and research closely monitored to promptly address and resolve issues. 
If a researcher knowingly acts in conflict with Oxfam's Code of Conduct, the research must be stopped and, depending on the action, a human resources or anti-corruption staff member may need to be involved. Oxfam's safeguarding focal point must be involved to investigate and take appropriate action. Oxfam GB's Reporting Misconduct Standard Operating Procedures provide clear guidelines on how to report suspicions of sexual, financial or human resources misconduct by staff.

In addition to aspects of safety, welfare and conduct, there are often glaring power asymmetries between locally based staff who carry out research and the funders of research, especially when they are international staff. These asymmetries can make research unethical. These include, for example, not sharing research findings with partners or local contacts and researchers, or monopolizing the resultant publications without involving or acknowledging the researchers that made it possible. These imbalances are increasingly recognized as a problem throughout development research (Kaplan et al. 2020). Ethical research acknowledges the role that people play in the most context-appropriate way.

\section{USING AND ADAPTING THIS GUIDELINE}

Oxfam operates as a confederation of independent charitable organizations in different countries. This document outlines the minimum standards of ethical research that should serve as a guide to any Oxfam staff member conducting research, including with partners and consultants. It can be used as a reference by other NGOs, community organizations and researchers working in fragile contexts and with vulnerable communities.

When context-specific and/or more detailed guidance is provided by Oxfam affiliates or their partners, researchers must adhere to the relevant protocols and procedures and demonstrate that they have done so.

The complexity of Oxfam's work, with research being conducted by multiple stakeholders across different countries, demands both pragmatism and agility. For example:

- All dimensions of ethical research, including upfront risk analyses, should be determined with locally based staff and any partners before research goes ahead to ensure maximum contextual relevance.

- Different stakeholders usually have different ethical protocols. These should be implemented in conjunction with each other, rather than reverting back to only one side's set of guidelines.

- Local staff know best what procedures and standards are realistic, and where they need to be adapted, for example around informed consent for groups with low literacy. Working with such constraints requires pragmatism, yet must not entail dropping ethical standards.

- Language and culture are closely interlinked with ethical research standards. For example, training workshops - which set the standard for the expected level of ethical practice for enumerators - have to be carefully tailored to each context. This necessitates trust and the delegation of shared ethical standards to trusted partners.

- It is the responsibility of researchers to gain a thorough understanding of how power dynamics (e.g. gendered conflict dynamics) and conflict dynamics - in general and more specifically in the context they are working in - play out during a research process and can negatively affect participants. It is also their responsibility to determine how not to perpetuate negative power dynamics while ensuring quality data collection. 


\section{Different approaches to research ethics}

These guidelines are based on ethical research standards that have emerged from the academic tradition, but acknowledge that Oxfam's mission and practice mean the organization needs to go above and beyond.

Academia typically operates with a clear set of standards for ethical research, which are reviewed and signed off by a university or departmental ethics review board. This review process is an integral part to research being allowed to proceed - but it is often one of the very few ways in which ethical research practices are measured in this space, leaving a host of problematic practices to go unchecked (Humphreys 2015).

Oxfam operates in a different space. For example, the organization-wide practice of reviewing research to ensure ethical standards will be met is less homogenized than in academia. Instead, the aim is to review and sign off risk analyses with diverse internal stakeholders. Oxfam has committed to decolonizing research practices, so avoiding harmful power imbalances is integral for research being allowed to continue, as is including all stakeholders under our duty of care. Our code of conduct must not just be signed upfront, but actually 'lived', with safeguarding and whistleblowing processes in place and accessible to all participants - and all of this must be closely monitored. These standards need to be continuously checked, updated and improved.

\section{ADDITIONAL RESOURCES}

If you have concerns or queries about particular ethical issues in Oxfam's research, please contact research@oxfam.org.uk for further advice.

As noted above, this guideline is based in part on the Framework for Research Ethics produced by the ESRC, which provides the basic standards for UK-funded social science research.

Additional resources include the following:

- The Social Research Association. (n.d.). A Code of Practice for the Safety of Social Researchers. https://the-sra.org.uk/common/Uploaded\%20files/SRA-safety-code-ofpractice.pdf

- British Sociological Association. (2017). Statement of Ethical Practice. https://www.britsoc.co.uk/media/24310/bsa statement of ethical practice.pdf

- $\quad$ R. Wiles, G. Crow, S. Heath and V. Charles. (2006). Anonymity and Confidentiality. http://eprints.ncrm.ac.uk/423/1/0206 anonymity\%2520and\%2520confidentiality.pdf

- Department for International Development. (2019). DFID Ethical Guidance for Research, Evaluation and Monitoring Activities. https://www.gov.uk/government/publications/dfid-ethicalguidance-for-research-evaluation-and-monitoring-activities

- Oxfam. (n.d.). Safeguarding policy and procedures at Oxfam. https://www.oxfam.org/en/whatwe-do/about/safeguarding

- Oxfam. (n.d.). Research guidelines. https://policy-practice.oxfam.org.uk/ourapproach/research/research-guidelines 


\section{REFERENCES}

Grimm, J., Koehler, K., Lust, E. M., Saliba, I., Schierenbeck, I. (2020). Chapter 3: In the Field. In Safer Field Research in the Social Sciences: A Guide to Human and Digital Security in Hostile Environments. SAGE Publications.

Humphreys, M. (2015). Reflections on the Ethics of Social Experimentation. Journal of Globalization and Development 6(1): 87-112. https://doi.org/10.1515/igd-2014-0016

Kaplan, L., Kuhnt, J. and Steinert, J.I. (2020) Do no harm? Field research in the Global South: Ethical challenges faced by research staff. World Development 127.

https://doi.org/10.1016/i.worlddev.2019.104810

McDermott, R., Gibbons, P., Mpanje, D., McGrath, S., Market, A., Cortés Ferrandez, P., Haryati, D. And Azizah, N. (2019). Beyond ethical approval: fostering ethical research practices within inter-sectoral research projects involving academic and NGO sector partners. Journal of International Humanitarian Action 4(18). https://doi.org/10.1186/s41018-019-0065-8

Way, E. (2013). Understanding Research Fatigue in the Context of Community-University Relations. Masters thesis, Clark University.

https://commons.clarku.edu/cgi/viewcontent.cgi?article=1018\&context=localknowledge

\section{LINKS}

All links last accessed September 2020.

UK Economic and Social Research Council (ESRC) Framework for Research Ethics:

https://esrc.ukri.org/funding/guidance-for-applicants/research-ethics/

Writing Terms of Reference for Research: https://policy-

practice.oxfam.org.uk/publications/writing-terms-of-reference-for-research-253034

Terms of Reference for Research Template: https://policypractice.oxfam.org.uk/publications/terms-of-reference-for-research-template-253035

Integrating Gender in Research Planning: https://policy-

practice.oxfam.org.uk/publications/integrating-gender-in-research-planning-620621

Doing Research with Enumerators: https://policy-practice.oxfam.org.uk/publications/doingresearch-with-enumerators-620574

UK ESRC Research Ethics, Our core principles: https://esrc.ukri.org/funding/guidance-forapplicants/research-ethics/our-core-principles/

Oxfam Policy \& Practice, research reports: https://policypractice.oxfam.org.uk/publications/search?i=1;q1=Publications; $q 2=$ Research+report;show $a l l=p r$ of;sort=publication date; $x 1=$ page type; $x 2=$ publication type

Democracy in Africa, How can we make research in the "Global South" more ethical? http://democracyinafrica.org/can-make-research-ethical/ 
UK ESRC, What if it is not possible to obtain informed consent?

https://esrc.ukri.org/funding/guidance-for-applicants/research-ethics/frequently-raised-

questions/what-if-it-is-not-possible-to-obtain-informed-consent/

Oxfam's Staff Code of Conduct: https://www.oxfam.org.uk/what-we-do/about-us/plans-reports-

and-policies/code-of-conduct

Going Digital: Improving data quality with digital data collection: https://policy-

practice.oxfam.org.uk/publications/going-digital-improving-data-quality-with-digital-data-collection$\underline{620522}$

World Health Organization, templates for informed consent forms:

https://www.who.int/ethics/review-committee/informed consent/en/

University of Manchester, 'Informed Consent' on Preparing an ethics application:

https://www.staffnet.manchester.ac.uk/rbe/ethics-integrity/ethics/app-prep/

University of Michigan, Informed Consent Guidelines \& Templates: https://research-

compliance.umich.edu/informed-consent-guidelines

UK Data Service, Consent for data sharing: https://www.ukdataservice.ac.uk/manage-data/legal-

ethical/consent-data-sharing/consent-forms

Oxfam's Ethical Content Guidelines https://policy-practice.oxfam.org.uk/publications/ethicalcontent-guidelines-upholding-the-rights-of-the-people-in-the-pictures-i-620935

Oxfam ‘One Oxfam Digital Safeguarding Policy' on Plans, reports and policies:

https://www.oxfam.org.uk/documents/49/OneOxfamDigitalSafeguardingPolicy EN.pdf

Oxfam ‘One Oxfam Youth Safeguarding Policy' on Plans, reports and policies:

https://www.oxfam.org.uk/documents/46/OneOxfamYouthSafeguardingPolicy EN.pdf

UK Data Service, Anonymisation: https://www.ukdataservice.ac.uk/manage-data/legalethical/anonymisation.aspx

European Data Protection Supervisor - Data Protection: https://edps.europa.eu/dataprotection/data-protection en\#Privacy

United Nations (UN) Universal Declaration of Human Rights: https://www.un.org/en/universaldeclaration-human-rights/

European Union (EU) Charter of Fundamental Rights of the European Union: https://eurlex.europa.eu/legal-content/EN/TXT/?uri=CELEX:12012P/TXT

EU Regulation 2016/679 of the European Parliament and of the Council [General Data Protection Regulation]: https://eur-lex.europa.eu/legal-content/EN/TXT/PDF/?uri=CELEX:32016R0679

Data Protection Act 2018, c12: https://www.legislation.gov.uk/ukpga/2018/12/contents/enacted

UN Conference on Trade and Development, Data Protection and Privacy Legislation Worldwide: https://unctad.org/en/Pages/DTL/STI and ICTs/ICT4D-Legislation/eCom-Data-Protection-

Laws.aspx 
Oxfam GB Data Protection Policy:

https://oxfam.app.box.com/s/e253mb6gk4jbt1ibndn5aluqq3secxyt

Responsible Data at Oxfam: Translating Oxfam's Responsible Data Policy into practice, two years on: https://policy-practice.oxfam.org.uk/publications/responsible-data-at-oxfam-translatingoxfams-responsible-data-policy-into-pract-620257

Safeguarding at Oxfam: https://www.oxfam.org.uk/about-us/tackling-abuse-information-andupdates/keeping-people-safe/

Oxfam GB 'Reporting Misconduct Standard Operating Procedures (SOP)'

https://www.oxfam.org.uk/documents/27/OGB Reporting Misconduct SOP.pdf 
(c) Oxfam International November 2020.

This guideline has been prepared by the Oxfam Research Network for use by development practitioners and researchers, including Oxfam staff and partners. It was written by Franziska Mager and Silvia Galandini, with thanks to Irene Guijt, Simone Lombardini, Jaynie Vonk, Emily Tomkys Valteri, James Eaton-Lee and Xara Church for their inputs.

The text may be used free of charge for the purposes of education and research, provided that the source is acknowledged in full. The copyright holder requests that all such use be registered with them for impact assessment purposes. For copying in any other circumstances, or for re-use in other publications, or for translation or adaptation, permission must be secured and a fee may be charged. Email publish@oxfam.org.uk.

Oxfam welcomes comments and feedback on its Research Guidelines. If you would like to discuss any aspect of this document, please contact research@oxfam.org.uk. For further information on Oxfam's research and publications, please visit

www.oxfam.org.uk/policyandpractice

The information in this publication is correct at the time of going to press.

Published by Oxfam GB for Oxfam International under ISBN 978-1-78748-641-6 in November 2020. DOI: $10.21201 / 2020.6416$

Oxfam GB, Oxfam House, John Smith Drive, Cowley, Oxford, OX4 2JY, UK. Oxfam is a registered charity in England and Wales (no 202918) and Scotland (SC039042).

Oxfam is an international confederation of 20 organizations networked together in 67 countries, as part of a global movement for change, to build a future free from the injustice of poverty.

www.oxfam.org 